\title{
41. On the Reflection of Electromagnetic Waves from a Meteor.
}

\author{
By Toshiyuki Yonezawa. \\ Physical Institute for Radio Waves, KoJaira-machi, Tokyo. \\ (Comm. by Y. HagihaRA, M. I. A., July, 12, 1946.)
}

It has already been experimentally ascertained both in our country and foreign lands that a meteor may reflect electromagnetic waves. This is of course not because electromagnetic waves are reflected by a meteoric body itself, but because a large number of ions that are produced along the path of a meteor on account of its ionizing effect make the values of the dielectric constant in the neighbourhood temporarily very small. But as it seems that no detailed calculation concerning it has ever been published, we shall here state some results of our own calculations.

For mathematical simplicity the following assumptions have been made. At the time $t=0$ a meteor shoots in a straight line and owing to its ionizing effect there leave behind it $N$ electrons per unit length along the line. If they have no velocity component perpendicular to the path of the meteor and spread out gradually by diffusion, the electron density at the point $r \mathrm{~cm}$ distant from the path of the meteor at the time $t=t$ is given by $\frac{N}{4 \pi D t} \exp$ $\left(-\frac{r^{2}}{4 D t}\right)$, where $D$ is the diffusion coefficient of an electron.(1) Besides diffusion electrons are also lost by recombination and attachment, but, as these effects are not so important as diffusion at least for a first few moments when electron density gradient is very great, they are neglected, and this is also necessary to avoid mathematical complexity. Thus the dielectric constant $\varepsilon$ at the same point is by the well-known formula

$$
\varepsilon=I-\frac{e^{2} N}{m \omega^{2} D t} e^{-\frac{r^{2}}{4 D t},}
$$

where $e, m$ and $\omega$ are respectively the charge on an electron in e.s.u., the mass of an electron and the angular frequency of the electromagnetic wave used. In the expression (1) the Lorentz polarization-correction is neglected, but when $\omega$ is not too large, there seems to be no very great influence upon the results.

Here the simplest case will be treated, i.e. we assume that the electromagnetic wave is a plane wave and its electric vector is perpendicular to the

(1) Cf., for instance, Kennard, Kinetic theory of gases (1938), pp. 280-287. 
path of the meteor. When such a wave is incident upon a space, where the distribution of the values of the dielectric constant is given by (I), from any direction perpendicular to the path of the meteor, one can get the asymptotic solution of the wave equation at sufficiently great distances and find the ratio $R$ of the reflected energy to the incident energy (we shall call this ratio " eflection coefficient"). At the point $r \mathrm{~cm}$ distant from the path of the meteor this is given by the following expression:

$$
\left.\begin{array}{rl}
R & =\frac{2}{\pi k r}\left|\frac{1}{2}\left(e^{-2 i \eta_{0}}-1\right)+\sum_{n=1}^{\infty}(-1)^{n}\left(e^{-2 \eta_{n}}-1\right)\right|^{2} \\
& =\frac{2}{\pi k r}\left[\left\{\frac{1}{2}\left(\cos 2 \eta_{0}-1\right)+\sum_{n=1}^{\infty}(-1)^{n}\left(\cos 2 \eta_{n}-1\right)\right\}^{2}+\left\{\frac{1}{2} \sin 2 \eta_{0}\right\}\right. \\
& \left.\left.+\sum_{n=1}^{\infty}(-1)^{n} \sin 2 \eta_{n}\right\}^{2}\right],
\end{array}\right\}
$$

where $k$ is $\omega$ divided by the light velocity in vacuo and $\eta_{n}$ is a constant that depends on $n$ and is defined in the following way. Let $L_{n}(r)$ be the solution of the differential equation

$$
\frac{d^{2}}{d r^{2}} L_{n}(r)+\frac{1}{r} \frac{d}{d r} L_{n}(r)+\left(k^{2}-\frac{n^{2}}{r^{2}}-\frac{e^{2} N}{m c^{2} D t} e^{-\frac{r^{2}}{4 \mathrm{D} t}}\right) L_{n}(r)=0 \text {, (n: integer) }
$$

that is regular at the origin; then at sufficiently great distances $L_{n}(r)$ has the following asymptotic form:

$$
L_{n}(r) \sim \text { const. } \sqrt{\frac{2}{\pi k r}} \cos \left(k r-\frac{2 n+1}{4} \pi+\eta_{n}\right) .
$$

The method of derivation of the expression (2) is almost the same as in the case of the scattering of a beam of particles by a centre of force treated in quantum mechanics. ${ }^{(2)}$ Only the problem is there three dimensional, whereas here it is two dimensional and corresponingly there is a few slight differences; for instance, Legendre's polynomials do not appear in the present case.

The formula (2) is exact, but the values of $\eta_{n}$ 's can in general only be determined by numerical integration and this is very troublesome. So using Jeffreys' approximation, ${ }^{(3)}$ we can find the approximate values of $\eta_{n}$; that is,

$$
\eta_{n}=\frac{n}{2} \pi-x_{0}+\int_{x_{0}}^{\infty}\left\{\left(1-\frac{n^{2}-\frac{1}{4}}{x^{2}}-\frac{e^{2} N}{m \omega^{2} D t} e^{-\frac{x^{2}}{4 k^{2} \mathrm{D} t}}\right)^{\frac{1}{2}}-1\right\} d x,
$$

where $x_{0}$ is the positive maximum zero-point of the function

$$
1-\frac{n^{2}-\frac{1}{4}}{x^{2}}-\frac{e^{2} N}{m \omega^{2} D t} e^{-\frac{x^{2} \cdot}{4 k^{2} \mathrm{D} t}}
$$

Using the values of $\eta^{\prime}{ }_{n}$ s given by (3) and examining the terms. of the series in the expression (2), we can see that the terms in the neighbourhood of $\boldsymbol{n}=$

(2) Cf. Mott \& Massey, Theory of atomic collisions, (1933), pp. 19-24.

(3) Cf. Mott \& Massey, l.c., pp. 90-94. 
0 mostly contribute to the sum of the series and other terms may be neglected. So we expand $\eta_{n}$ in a power series of $n$ and neglect higher terms than $n .^{2}$ Substituting this in (2) and we replace the sum of the series by the corresponding integral. Thus we get for the reflection coefficient $R$ the following formula :

$$
R=\frac{1}{8 k r\left|\left[\frac{d^{2} \eta_{n}}{d n^{2}}\right]_{n=0}\right|}
$$

and from (3) we get

$$
\left.\left[\frac{d^{2} \eta_{n}}{d n^{2}}\right]_{n=0}=-\int_{x_{0}}^{\infty} \quad x^{2} \sqrt{1+\frac{1}{4 x^{2}}-\frac{e^{2} N}{m\left(\omega^{2} D \bar{t}\right.} e^{-\frac{x^{2}}{4 k^{2} D t}}}\right\}^{(4)}
$$

Using (4) in the cases of $N=\mathrm{I} .32 \times 10,{ }^{15} 0.88 \times 10^{15}$ and $0.44 \times 10^{15}$ we have calculated $8 k r R$ as a function of $4 k^{2} D t$. Fig. I is its graphical representation, taking $8 k r R$ as ordinate and $4 k^{2} D t$ as abscissa. The abscissa is proportional to time, but the constant of proportion depends on the wave-frequency used and the diffusion coefficient of an electron (in the neighbourhood of the point where the wave is reflected), so that, when we graduate the abscissa in time, the time-scale will be different corresponding to the different. values of frequency and diffusion coefficient. For instance, if the wave-frequency. is $6 \mathrm{Mc} /$ $\mathrm{sec}$ and the diffusion coefficient of an electron is $10^{6} \mathrm{~cm}^{2} / \mathrm{sec}$, we get the time-scale in Fig. I. The ordinate is proportional to the reflection coefficient $R$, but the constant of proportion depends on the wave-frequency and the distance to the path of the meteor. If their values are respectively $6 \mathrm{Mc}$ and $150 \mathrm{~km}$, we have the scale of reflection coefficient in the figure. Thus Fig. I indicates how the reflection coefficient changes with time, if we take suitable scales for the abscissa $t$ and ordinate $R$ corresponding to the respective cases. We can see in the figure that the intensity of reflection at first in-

Fig. 1.

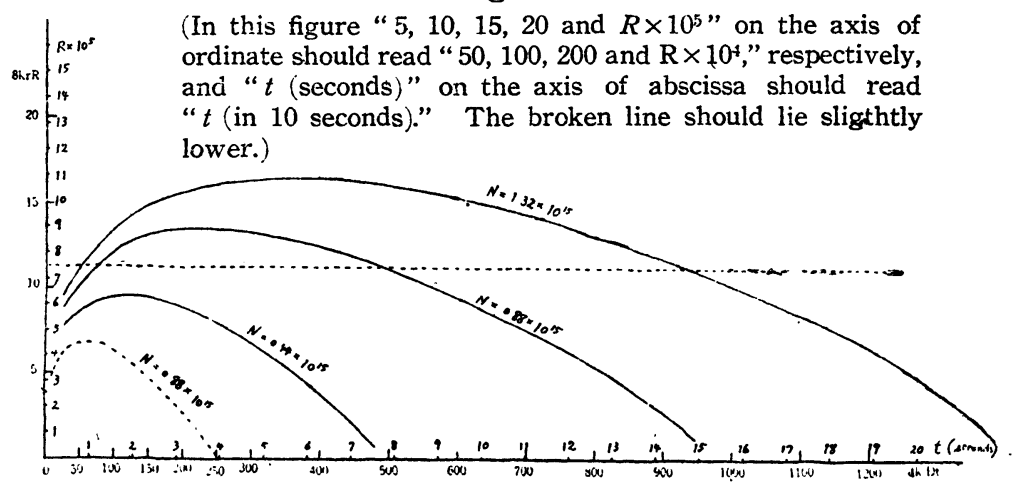


creases more rapidly and reaches the maximum value, and them decreases more slowly towards zero, and also thar the reflection coefficient increases with $N$ and the larger is the value of $N$, the later becomes the time of the maximum intensity. of reflection.

As an illustration we take the case that the power of the transmitter is I $\mathrm{kW}$ and the minimum perceptible electric field-intensity of the receiver is $0.3 \mathrm{microvolt} / \mathrm{cm}$. Then under the same conditions as the preceding paragraph. we get the value indicated by a broken line in Fig. 1 as the critical value of reflection coefficient in order that the reflection is perceptible. So if $N=0.44 \times 10^{15}$, we shall have no reflection and if $N=$ $0.88 \times 10^{15}$, the reflection will begin to appear about 7 seconds after the pass 0 : the meteor and will persist for about 84 seconds, and if $N=1.32 \times 10^{15}$, the time-lag and the duration of reflection will be about 5 second and 155 second: respectively. If the frequency becomes two times, the abscissa $4 k^{2} D t$ will br four times and so will be the time scale also. If we want to fix the time

Fig. 2.

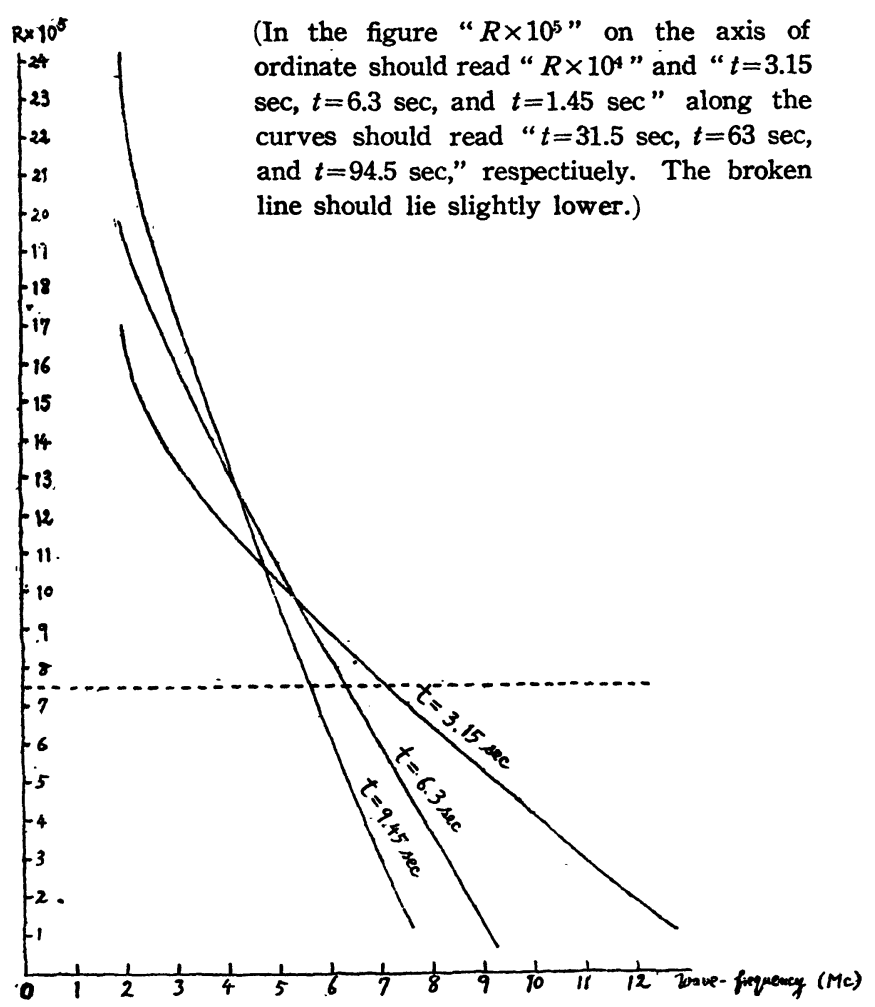


scale, we must reduce the breadth of the curve to-one-fourth. Also as the scale of the reflection coefficient $R$ becomes two times in that case, if we want to fix the scale of $R$, we must reduce the height of the curve by half. Thus for the frequency of I2 Mc we get the curve depicted in the lower left part of Fig. I in a broken line, if the scales of time and reflection coefficient are conserved, and under the above conditions no reflection will be perceived for $12 \mathrm{Mc}$.

Using this figure we have calculated the reflection coefficient $R$ as a function of frequency for the values of $t=31.5 \mathrm{sec}, 63 \mathrm{sec}$ and $94.5 \mathrm{sec}$ under the conditions $N=0.88 \times 10^{15}, r=150 \mathrm{~km}$ and $D=10^{6} \mathrm{~cm}^{2} / \mathrm{sec}$. Fig. 2 represents this graphically. We see that the reflection coeffcient. increases rapidly as the wave-frequency decreases and that this becomes more conspicuous as time passes.

Now we shall briefly compare these theoretical results with observations. For instance according to the observations of J.A. Pierce(4) the time-lag and the duration were respectively 16 seconds and I minute for a meteor of 2 magnitude and 24 seconds and 7.3 minutes for one of -1 magnitude in the case of the wave-frequency of $3.0 \mathrm{Mc}$ and the durations were 30 seconds and 1.5 minutes in the case of $6.4 \mathrm{Mc}$. This shows that both the time-lag and the duration are roughly in agreement with the theoretical expectation, but the difference of reflection due to that of frequency is not so remarkable as the theory. A number of observations that we have made also suggest almost the same tendency. In a private conversation with Mr. Tetuo Kohno (Electrotechnical Laboratory, Ministry of Communications) I was told that for $20 \mathrm{Mc}$ he had observed almost no time-lag and the duration had been at most a few seconds. In this case we must take the value of $N$ much larger than above in order to make the theoretical values of time-lag and duration consistent with observations. And as the quantitative investigation of the process of ionization is very difficult, we cannot say if such large values of $N$ are physically possible. But the observations cannot be said to have been carried out sufficiently, and in particular the path of a meteor is often not definitely determined, so that the complete comparison of the theory with observations is impossible. We wish to make detailed observations in future in order to check the above throry.

(4) Cf. Phys. Rev. 59 (1941), 625-626. 